\title{
The Effects of Exercise-induced Fatigue on Acetylcholinesterase Expression and Activity at Rat Neuromuscular Junctions
}

\author{
Guo Wen ${ }^{1,2}$, Wang Hui ${ }^{1}$, Chen Dan ${ }^{1}$, Wu Xiao-Qiong ${ }^{1}$, Tong Jian-Bin ${ }^{1}$, Li Chang-Qi ${ }^{1}$, \\ Lei De-Liang', Cai Wei-Jun', Li Zhi-Yuan' ${ }^{1}$ and Luo Xue-Gang1
}

${ }^{1}$ Department of Anatomy \& Neurobiology, Xiangya School of Medicine, Central South University, Changsha, Hunan 410013, China and ${ }^{2}$ Department of Physical Education, Hunan First Normal University, Changsha, Hunan 410012, China

Received May 24, 2009; accepted July 2, 2009; published online September 15, 2009

\begin{abstract}
Acetylcholinesterase is the enzyme that terminates neurotransmission by hydrolyzing the acetylcholine released by the motoneurons at the neuromuscular junctions. Although acetylcholinesterase has been studied for almost a century, the underlying relationship between exercise-induced fatigue and acetylcholinesterase activity at the synaptic cleft is not clear. The purpose of this study was to assess the effects of exercise-induced fatigue on the expression and activity of acetylcholinesterase at the neuromuscular junctions. The expression and activity of acetylcholinesterase at the gastrocnemius neuromuscular junctions was decreased transiently by exercise-induced fatigue and then gradually increased over $24 \mathrm{hr}$. The expression of acetylcholinesterase in the $24 \mathrm{hr}$ recovery group returned to the level of the control (non-exercised) group, but the activity of acetylcholinesterase remained significantly lower. These data suggest that the decrease of acetylcholinesterase expression and activity may be involved in the production and/or maintenance of exercise-induced fatigue.
\end{abstract}

Key words: exercise, central fatigue, peripheral fatigue, acetylcholinesterase, neuromuscular junctions

\section{Introduction}

Exercise-induced fatigue is a reduction in maximal voluntary muscle force that results from intense physical activity. It is traditionally divided into two distinct components: i) central fatigue, involving insufficient neural drive to the muscle, and ii) peripheral fatigue, including changes at the neuromuscular junctions (NMJs) $[7,8,13]$. The NMJs is the synapse between the presynaptic motor neurone and the postsynaptic muscle membrane. At mammalian NMJs, neurotransmission relies on the activity of key proteins such as acetylcholine (ACh), choline acetyltransferase (ChAT), acetylcholinesterase (AChE) and acetylcholine receptor (AChR). At the presynaptic neuron end, an electrical impulse triggers the release of $\mathrm{ACh}$, which accumulates

Correspondence to: Li Zhi-Yuan and Luo Xue-Gang, Department of Anatomy \& Neurobiology, Xiangya School of Medicine, Central South University, Changsha, 410013, China.

E-mail: lizhiyuan2000@yahoo.com (Li Zhi-Yuan); xgluo@xysm.net (Luo Xue-Gang) in vesicles into the synaptic cleft via exocytosis. ACh then binds to an ACh receptor (AChR) on the postsynaptic neuron surface, and the ACh-AChR binding induces subsequent impulses to the postsynaptic neuron. Finally, $\mathrm{ACh}$, which is released again by the receptor into the synaptic cleft, is rapidly degraded by AChE $[15,22]$.

In previous studies of exercise-induced fatigue, attention has been on pre-synaptic (ACh, ChAT) and post-synaptic (AChR) molecules. Less research has been directed toward investigating NMJs AChE and its potential role in fatigue. Previous studies show that the functional failure of motor muscles might be due to an accumulation of acetylcholine (ACh) in the synaptic cleft during high-frequency synaptic activity that eventually results in a failure to generate muscle action potentials [18]. In the present study, we investigate the expression and activity of AChE in the skeletal muscle of exercise-fatigued rats using immunohistochemistry, histochemistry, enzyme linked immunoabsorbent assay and chemistry colorimetric method. We hypothesized that AChE expression and activity have roles in producing or maintaining exercise-induced fatigue. 


\section{Material and Methods}

\section{Animals}

All animals received care compliant with the "Guide for the Care and Use of Laboratory Animals," published by the National Institutes of Health (NIH publication 85-23, revised 1986). Briefly, 60 healthy adult male Sprague-Dawley rats (8 weeks old), weighing 200-250 g, were singly housed on a 12:12 hr light-dark cycle in a low-stress environment $\left(22^{\circ} \mathrm{C}, 50 \%\right.$ humidity, and low-noise) and received tap water and standard rat chow ad libitum. All the experiments were performed at the end of the active dark cycle.

\section{Exercise protocol}

Animals were randomly divided into one of six groups. Each group was composed of 10 animals (the right gastrocnemius of 5 animals for immunohistochemistry, the right gastrocnemius of 5 animals for histochemistry, and the left gastrocnemius of all 10 animals for enzyme linked immunoabsorbent assay and chemistry colorimetric method). One group was a control group (non-exercised) $(n=10)$ and the remaining five groups were exercised to fatigue with the following recovery times: $0 \mathrm{hr}$ (no recovery) $(\mathrm{n}=10), 3 \mathrm{hr}$ $(\mathrm{n}=10), 6 \mathrm{hr}(\mathrm{n}=10), 12 \mathrm{hr}(\mathrm{n}=10)$, and $24 \mathrm{hr}(\mathrm{n}=10)$. Exercise training was done on motorized rodent treadmills (SLYRTML six-lane treadmill for rat, Beijing). Exercised rats ran to exhaustion at $28 \mathrm{~meters} / \mathrm{min}$. Rats were gently handprodded occasionally to maintain running status. Fatigue was defined as the point when the rats failed to maintain pace with the treadmill despite 2 min of gentle prodding with the hand [24]. After reaching fatigue, 0 hour group rats were sacrificed immediately, and runners from $3 \mathrm{hr}$, $6 \mathrm{hr}, 12 \mathrm{hr}$, and $24 \mathrm{hr}$ recovery groups were returned to their cages where they were given food and water ad libitum for their specified recovery times. Control group rats remained in their cages in the treadmill room. All rats were acclimated to treadmill running a minimum of two times per day at $10 \mathrm{~meters} / \mathrm{min}$ for $10 \mathrm{~min}$ for 3 days before the actual fatigue experiment.

\section{AChE expression and activity}

For histochemistry, animals were anesthetized with $2 \%$ pentobarbital $(40 \mathrm{mg} / \mathrm{kg})$ and perfused transcardially with $0.9 \%$ sodium chloride, followed by $4 \%$ paraformaldehyde in phosphate buffer $(0.1 \mathrm{M}, \mathrm{pH} 7.4)$. After perfusion, the gastrocnemius was dissected out and post-fixed in $4 \%$ paraformaldehyde for $2 \mathrm{hr}$ at room temperature, then dehydrated by gradual immersion in $15 \%$ sucrose phosphate buffer for $4 \mathrm{hr}$ at $4{ }^{\circ} \mathrm{C}$ and $30 \%$ sucrose phosphate buffer overnight at $4^{\circ} \mathrm{C}$ [2]. Longitudinal $35 \mu \mathrm{m}$-thick sections were cut on a cryostat. The sections were collected on gelatin-coated glass slides and dried overnight at room temperature. After washing two times with acetic acid buffer $(0.1 \mathrm{M})$, the sections were incubated for $1 \mathrm{hr}$ at $37^{\circ} \mathrm{C}$ for the demonstration of $\mathrm{AChE}$ in motor endplates as described by Karnovsky and Roots [11] with modifications by Gruber and Zenker [9] in a solution containing acetylthiocholine iodide $(5 \mathrm{mg})$, acetic acid buffer $(6.5 \mathrm{ml}$, $0.1 \mathrm{~mol} / \mathrm{L}, \mathrm{pH} 5.5)$, sodium citrate $(0.5 \mathrm{ml}, 0.1 \mathrm{~mol} / \mathrm{L})$, cupric sulfate $(1 \mathrm{ml}, 30 \mathrm{mmol} / \mathrm{L})$, distilled water $(1 \mathrm{ml})$, and kalium ferricyanatum $(1 \mathrm{ml}, 5 \mathrm{mmol} / \mathrm{L})$. The sections were then rinsed with distilled water and coverslipped with glycogelatin.

For AChE expression immunohistochemistry and the demonstration of $\mathrm{AChE}$ in motor endplates as described in the literature $[6,12]$, animals were prepared as aforementioned without sucrose immersion, then the gastrocnemius rinsed three times in phosphate buffered saline (PBS). Longitudinal sections $250 \mu \mathrm{m}$-thick were cut on a cryostat. The sections were washed three additional times with PBS, then soaked for $20 \mathrm{~min}$ in $0.3 \% \mathrm{H}_{2} \mathrm{O}_{2}$ and methanol in PBS to inactivate endogenous peroxidase activity. After the elimination of non-specific antibody binding in 5\% bovine serum in PBS, the sections were incubated with a goat anti-AChE polyclonal antibody (Santa Cruz Biotechnology, Inc., Santa Cruz, CA) at a dilution of $1: 200$ at $4{ }^{\circ} \mathrm{C}$ overnight, then with a biotinylated anti-goat IgG at a dilution of 1:200 (Vector Laboratories Inc., Burlingame, CA) for $2 \mathrm{hr}$ at $37^{\circ} \mathrm{C}$. After incubation with avidin-biotin complex $(\mathrm{ABC}$, Vector Laboratories Inc., Burlingame, CA) for $2 \mathrm{hr}$ at $37^{\circ} \mathrm{C}$, the sections were visualized following incubation in a PBS solution containing $0.05 \% \mathrm{DAB}$ and $0.03 \% \mathrm{H}_{2} \mathrm{O}_{2}$ for $10 \mathrm{~min}$ at room temperature. Finally, the sections were dehydrated, cleared, and coverslipped. In order to exclude false positive results, control sections were processed in exactly the same way replacing the primary antibody with normal goat serum.

For quantification of $\mathrm{AChE}$ expression and activity in muscle, animals were anesthetized with $2 \%$ pentobarbital $(40 \mathrm{mg} / \mathrm{kg})$. Small strips of the innervated region $(5 \times 5 \mathrm{~mm})$ of the gastrocnemius were cut, then homogenized and centrifuged $\left(3000 \times \mathrm{g}\right.$ at $4^{\circ} \mathrm{C}$ for $\left.10 \mathrm{~min}\right)$. The supernatants were stored at $-20^{\circ} \mathrm{C}$ for further analyses. Then, the animals were perfused transcardially with $0.9 \%$ sodium chloride, followed by $4 \%$ paraformaldehyde in phosphate buffer for $\mathrm{AChE}$ histochemistry and immunohistochemistry. AChE concentration were analyzed according to the enzyme linked immunoabsorbent assay provided with the commercial kit (E1447r, Wuhan Uscn Sciences Co. Ltd., China). AChE activity were analyzed according to the standard spectrophotometric procedures provided with the commercial kit (A024, Nanjing Jiancheng Institute of Biotechnology, China) using a Shanghai 722 (Shanghai, China) spectrophotometer.

\section{Data analysis}

Five animals and four sections from each experimental animal were used for data collection in both the histochemical and immunohistochemical experiments. Each section was photographed in eight visual fields under a Motic 5000 microscope, hence the sample number was 5 animals $\times 4$ sections $\times 8$ fields. Pictures were analyzed with Motic Images Advanced 3.2 system. In this system, the average gray value from black to white ranged from 0 to 255 . In the present study, we calculated the parameter "gray value" as the difference between average gray value of the background and 
that of the positive products, such that a higher gray value indicates a lower positive product, or AChE. The parameter "area of positive products" is the area of stained structures relative to the area of background (we chose the wholetissue area as the background reference area) in histochemistry and immunohistochemistry.

For the statistical analysis of the quantitative experiments, 10 samples of each group were used. AChE concentrations were calculated and standardized based on protein concentrations of the gastrocnemius samples. The values for the AChE concentrations were calculated according to the formula generated from the trend line of the standard curve. The value was then multiplied by the dilution factor to generate the final value for AChE concentration. The values for $\mathrm{AChE}$ activity were calculated according the formula given in the operating instructions.

All data were analyzed using commercial software (SigmaStat, SPSS, Chicago, IL), and expressed as the means \pm SD. One way-ANOVA was used for statistical analysis, Post-hoc comparisons were performed for each recovery time group vs. control group and p-values $<0.05$ being considered significant.

\section{Results}

\section{AChE expression}

We used AChE immunohistochemistry and enzyme linked immunoabsorbent assay to investigate expression changes at NMJs of the gastrocnemius at different recovery times after exercise-induced fatigue. As shown in Figure 1,
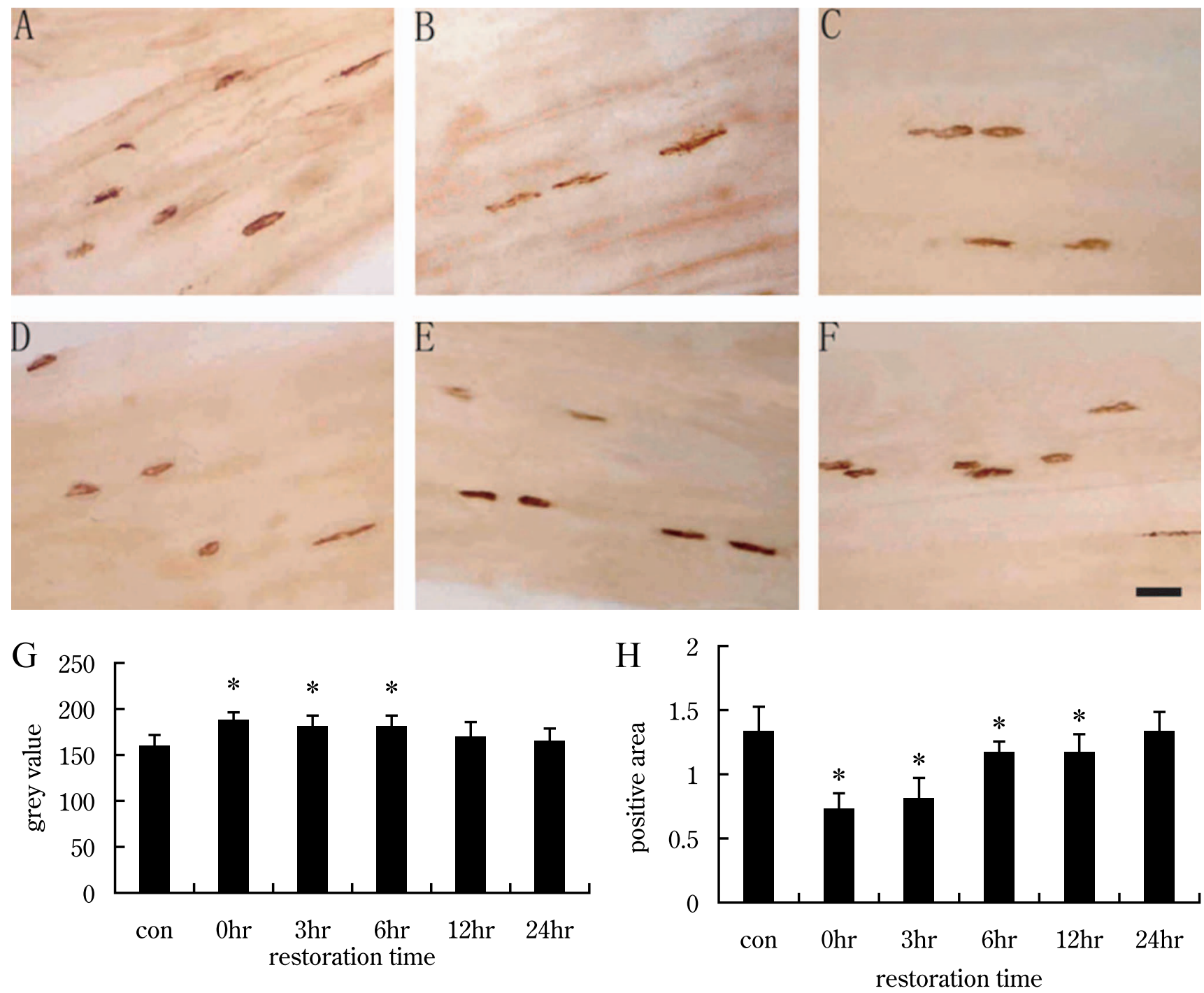

Fig. 1. AChE immunohistochemistry of gastrocnemius at different recovery times after exercise-induced fatigue. Bar=50 $\mu$ m. A: Control group. B-F: Groups with recovery times $0,3,6,12$ and $24 \mathrm{hr}$ after exercise-induced fatigue. G: Analysis of the gray value of AChE positive products in the control group and recovery groups. H: Analysis of the area of AChE positive products, relative to background area. The expression of AChE was decreased at $0 \mathrm{hr}$ after exercise-induced fatigue $(\mathbf{B}, \mathbf{G}, \mathbf{H})$, then gradually increased $(\mathbf{C}-\mathbf{E}, \mathbf{G}, \mathbf{H})$, and to control group levels in the $24 \mathrm{hr}$ recovery group $(\mathbf{F}, \mathbf{G}, \mathbf{H}) .{ }^{*} \mathrm{p}<0.05$ vs control group. 
AChE staining was brownish-red in color, and AChE immunopositive products with dot-like structures were detected at motor endplate regions in the gastrocnemius (Fig. 1A-F). After exercise-induced fatigue, AChE expression decreased, as indicated by increased gray value (Fig. $1 \mathrm{G}$ ), then gradually increased (Fig. 1G). At the recovery times of $12 \mathrm{hr}$ and $24 \mathrm{hr}$, the expression of AChE returned to control levels (Fig. 1G). The "area of positive products," relative to background area, as well as the AChE concentration (pg/mg), decreased at fatigue (recovery $0 \mathrm{hr}$ ) (Fig. 1H, Fig. 3), then gradually increased during recovery (Fig. 1H, Fig. 3). In the $24 \mathrm{hr}$ group, the "area of positive products" and the concentration of AChE returned to control level (Fig. 1H, Fig. 3).

\section{AChE activity}

To detect AChE activity, we employed AChE histochemical staining and chemistry colorimetric method. As shown in Figure 2, AChE activity was detected at motor endplate regions in gastrocnemius. The area of active AChE is elliptical or orbicular-ovate, reflective of the motor endplate. After exercise-induced fatigue, the positive staining of AChE was faded (Fig. 2B), then gradually became dark again with recovery (Fig. 2C-F), suggestive of greater activity. This finding was supported by gray value and area analyses. The "gray value" of AChE increased at time $0 \mathrm{hr}$ after fatigue, indicating low AChE activity. Gray value then gradually decreased, indicating higher AChE activity. In
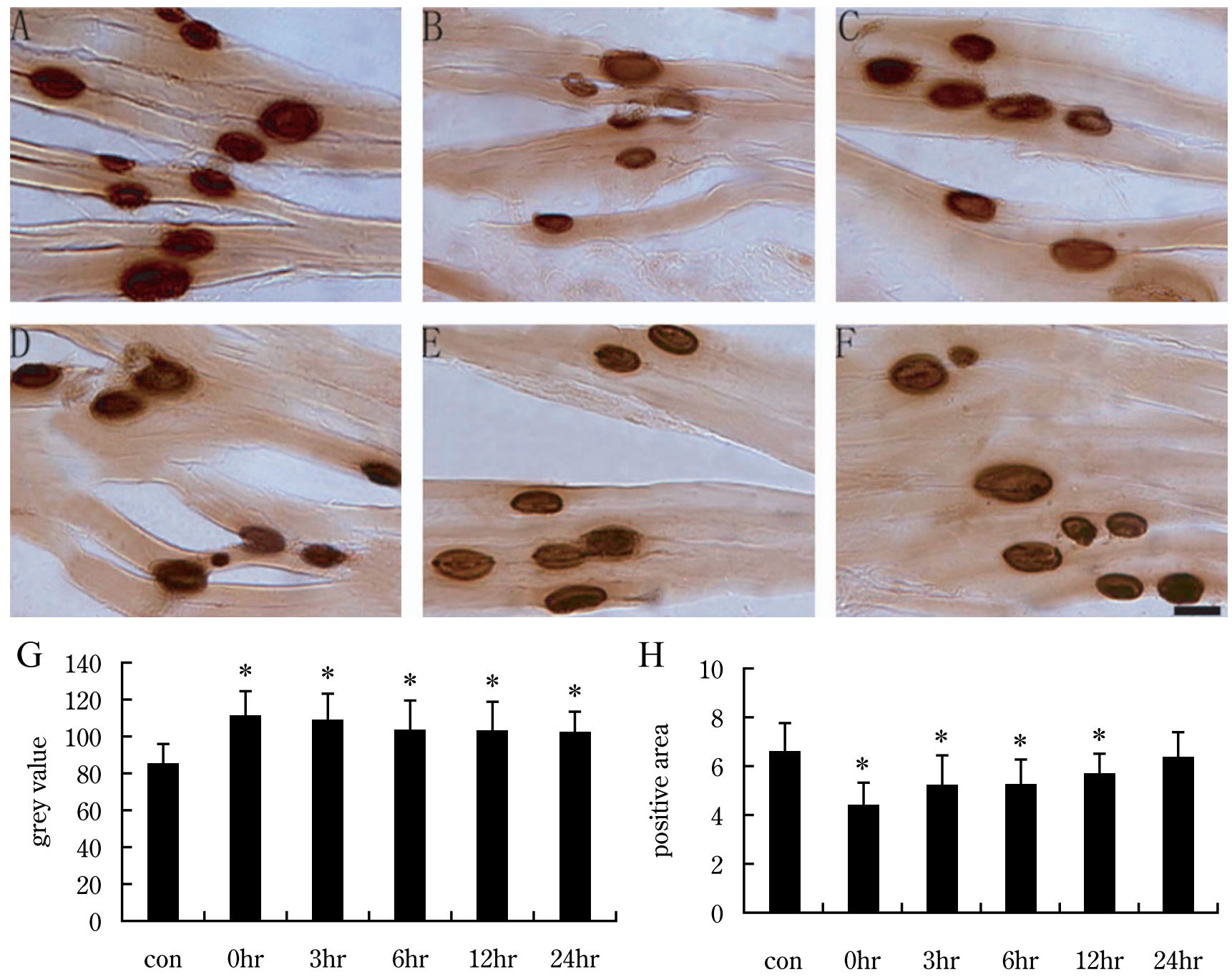

restoration time

restoration time

Fig. 2. AChE histochemistry of gastrocnemius at different recovery times after exercise-induced fatigue. Bar=50 $\mu \mathrm{m}$. A: Control group. B-F: Groups with recovery times 0, 3, 6, 12 and $24 \mathrm{hr}$. G: Analysis of the gray value of AChE positive products in control and recovery groups. H: Analysis of the area of AChE positive products. The activity of AChE was decreased at 0 hour after exercise-induced fatigue $(\mathbf{B}, \mathbf{G}, \mathbf{H})$, then gradually recovered (Fig. 2C-E, G, H). At 24 hr post-fatigue, the AChE activity was still significantly less than that of the control. 


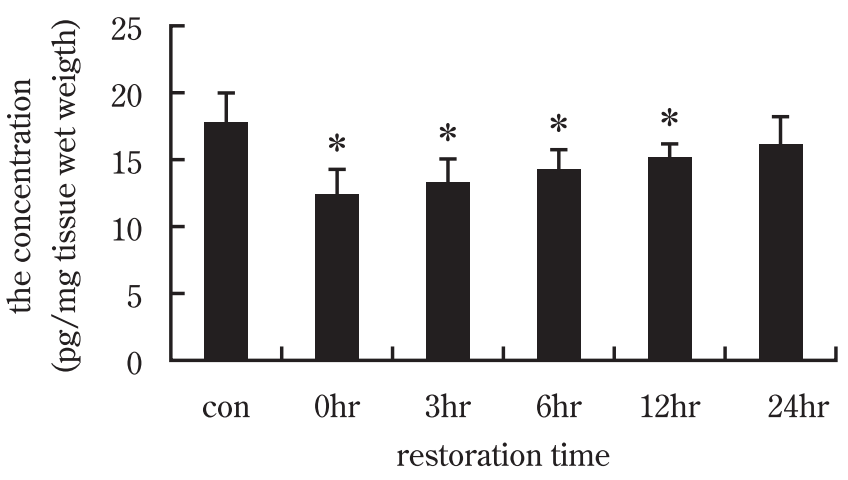

Fig. 3. Histograms show AChE concentration at different recovery times after exercise-induced fatigue with enzyme linked immunoabsorbent assay. AChE concentration was decreased at $0 \mathrm{hr}$ after exercise-induced fatigue, then gradually increased, and to control group levels in the $24 \mathrm{hr}$ recovery group.

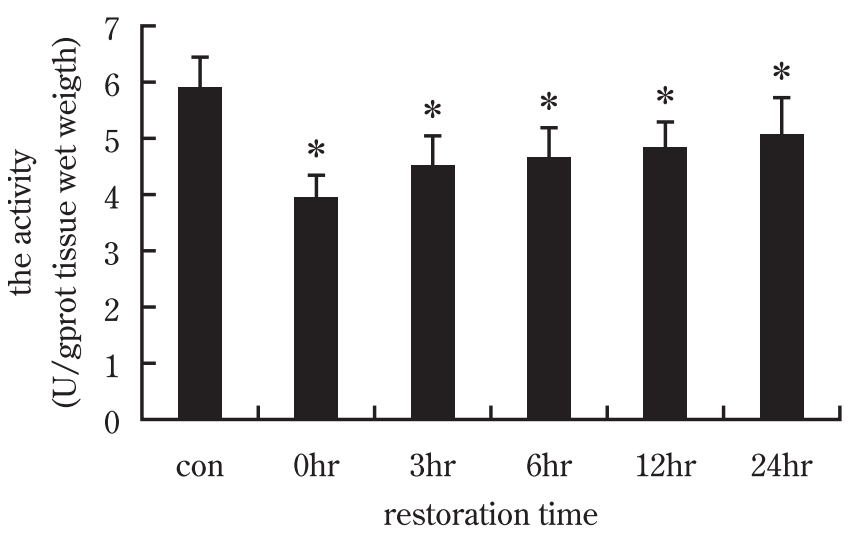

Fig. 4. Histograms show AChE activity at different recovery times after exercise-induced fatigue with chemistry colorimetric method. The activity of AChE was decreased at $0 \mathrm{hr}$ after exercise-induced fatigue, then gradually recovered. At $24 \mathrm{hr}$ post-fatigue, the activity of AChE was still significantly less than control.

the $24 \mathrm{hr}$ recovery group, the gray value of AChE was still significantly higher than control group, indicating a lower activity of AChE at $24 \mathrm{hr}$ than in control (p<0.05, Fig. 2G). The area of positive products and the activity of AChE (U/gprot) reached their minimum in the $0 \mathrm{hr}$ recovery group, then gradually increased in the other recovery groups (Fig. $2 \mathrm{H}$, Fig. 4). In the $24 \mathrm{hr}$ recovery group, the area of positive products of AChE returned to control level (Fig. 2H), but the activity of AChE was still significantly lower in the $24 \mathrm{hr}$ recovery group than control group (Fig. 4, $\mathrm{p}<0.05$ ).

\section{Discussion}

In the present study, our data show that exerciseinduced fatigue decreases $\mathrm{AChE}$ expression at the rats gastrocnemius NMJs. Specifically, we found that exerciseinduced fatigue generates a $30 \%$ decreased in AChE concentration, a $16 \%$ decrease in $\mathrm{AChE}$ positive staining (reflected by $16 \%$ increased "gray value"), and a $44 \%$ decrease in the AChE "area of positive products". AChE is an important component of all cholinergic synapses in the central and peripheral nervous systems, where it rapidly hydrolyzes acetylcholine released from the nerve terminals [23]. Previous studies suggest that the pattern of muscle activation is important for expression of AChE [21]. For example, in the extensor digitorum longus of the hindlimb suspended rat, a small but significant decrease in AChE mRNA has been observed [19]. Increased activation of the extensor digiorum longus muscle tends to suppress AChE mRNA [21]. We similarly show a decrease in AChE expression in gastrocnemius following exercise-induced fatigue.

It is well established that AChE activity is also influenced by the pattern of nerve impulses [14, 21], muscle electromechanical activity [20], and neurogenic substances conveyed by axonal transport [5]. The literature reveals that changes in neuromuscular activity lead to changes in muscle acetylcholinesterase activity $[3,10]$. In the present study, we found exercise-induced fatigue induced a roughly $33 \%$ depression in AChE activity (Fig. 2G, 2H, Fig. 4) in the gastrocnemius.

The current findings of exercise-induced reductions in AChE activity following fatigue are important when we consider the effects of AChE deficiency. Several studies have shown $\mathrm{AChE}$ absence in $\mathrm{AChE}^{-/-}$mice results in mild phenotypic abnormality (with mild movement and behavior, and reduced muscle weight, crosssectional area of muscle fibres and absolute maximal isometric force) [4, 25, 26]. Characteristics such as muscle weakness have also been reported in humans deficient in endplate AChE [17]. It has been well documented that a deficiency of AChE results in a change in hind limb muscle function and decreased ability of the hindlimb to resist fatigue [1]. Submaximal force potentiation, tetanic fade, and a very low fatigue resistance were also observed in 4- and 9-month-old AChE KO mice. Further, muscle weight, cross-sectional area of muscle, fibres and absolute maximal isometric force were also reduced in KO mice compared with WT mice [16].

Although the aforementioned investigations provide important evidence that the pattern of nerve impulses, muscle electromechanical activity, and neurogenic substances all influence AChE activity, and that the change in AChE is tied to changes in hind limb muscle function, the relationship between exercise-induced fatigue and NMJs AChE are not well known. Based on these previous studies and our current results, we believe that exercise-induced fatigue may induce continuous $\mathrm{ACh}$ release from the nerve terminals during strenuous physical exercise. The decrease of the AChE expression and activity observed would lead to an incomplete hydrolysis of the $\mathrm{ACh}$, and an accumulation of synaptic cleft ACh, slowing cholinergic reuptake. At the same time, repetitive nerve stimulation during exercise could induce a desensitization of the AChR.

In conclusion, we have shown that after exerciseinduced fatigue, the expression and activity of AChE is deceased at the rat gastrocnemius NMJs. Our results in- 
dicate that the recovery of AChE expression and activity are related to time and that expression recovers within 24 $\mathrm{hr}$, while activity is not quite fully restored at this time point. This study provides a starting point for further investigation of the mechanisms of AChE down-regulation due to exercise-induced fatigue.

\section{Acknowledgments}

This work was supported by a grant from the Scientific Research Fund of Hunan Provincial Education Department, China (No. 06C057).

\section{References}

1. Chau, D. T., Rada, P. and Kosloff, R. A. (2001) Nucleus accumbens muscarinic receptors in the control of behavioral depression: antidepressant-like effects of local M1 antagonist in the Porsolt swim test. Neuroscience 3; 791-798.

2. Chen, D., Tong, J. B., Wang, H., Zeng, L. P., Zhou, J., Huang, J. F. and Luo, X. G. (2008) Synaptophysin expression in rat retina following acute high intraocular pressure. Acta Histochem. Cytochem. 41; 173-178.

3. Collins, P. L. and Younkin, S. G. (1982) Effect of denervation on the molecular forms of acetylcholinesterase in rat diaphragm. $J$. Biol. Chem. 257; 13638-13644.

4. Duysen, E. G., Stribley, J. A., Fry, D. L., Hinrichs, S. H. and Lockridge, O. (2002) Rescue of the acetylcholinesterase knockout mouse by feeding a liquid diet; phenotype of the adult acetylcholinesterase deficient mouse. Dev. Brain Res. 137; 43-54

5. Fernandez, H. L., Patterson, M. R. and Duell, M. J. (1980) Neurotrophic control of $16 \mathrm{~S}$ acetylcholinesterase from mammalian skeletal muscle in organ culture. J. Neurobiol. 11; 557-570.

6. Fernandez, H. L., Ross, G. S. and Nadelhaft, I. (1999) Neurogenic calcitonin gene-related peptide: a neurotrophic factor in the maintenance of acetylcholinesterase molecular forms in adult skeletal muscles. Brain Res. 844; 83-97.

7. Gandevia, S. C. (2001) Spinal and supraspinal factors in human muscle fatigue. Physiol. Rev. 81; 172-189.

8. Garrandes, F., Colson, S. S., Pensini, M., Seynnes, O. and Legros, P. (2007) Neuromuscular fatigue profile in endurance-trained and power-trained athletes. Med. Sci. Sports Exerc. 39; 149-158.

9. Gruber, H. and Zenker, W. (1978) Acetylcholinesterase activity in motor nerve fibres in correlation to muscle fibre types in rat. Brain Res. 141; 325-334.

10. Gupta, R. C., Misulis, K. E. and Dettbarn, W. D. (1985) Changes in the cholinergic system of the rat sciatic nerve and skeletal muscle following suspension-induced disuse. Exp. Neurol. 89; 622-633.

11. Karnovsky, M. J. and Roots, L. (1964) A "direct-coloring" thiocholine method for cholinesterases. J. Histochem. Cytochem. $12 ; 219-221$.

12. Krejci, E., Legay, C., Thomine, S., Sketelj, J. and Massoulié, J. (1999) Differences in expression of acetylcholinesterase and collagen $\mathrm{Q}$ control the distribution and oligomerization of the collagen-tailed forms in fast and slow muscles. J. Neurosci. 19;
10672-10679

13. Lepers, R., Maffiuletti, N. A., Rochette, L., Brugniaux, J. and Millet, G. Y. (2002) Neuromuscular fatigue during a longduration cycling exercise. J. Appl. Physiol. 92; 1487-1493.

14. Lomo, T., Massoulie, J. and Vigny, M. (1985) Stimulation of denervated rat soleus muscle with fast and slow activity patterns induces different expression of acetylcholinesterase molecular forms. J. Neurosci. 5; 1180-1187.

15. Massoulié, J., Pezzementi, L., Bon, S., Krejci, E. and Vallette, F. M. (1993) Molecular and cellular biology of cholinesterases. Prog. Neurobiol. 41; 31-91.

16. Mouisel, E., Blondet, B., Escourrou, P., Chatonnet, A., Molgó, J. and Ferry, A. (2006) Outcome of acetylcholinesterase deficiency for neuromuscular functioning. Neurosci. Res. 55; 389-396.

17. Ohno, K., Engel, A. G., Brengman, J. M., Shen, X. M., HeidenReich, F., Vincent, A., Milone, M., Tan, E., Demirici, M., Walsh, P., Nakano, S. and Akiguchi, I. (2000) The spectrum of mutations causing end-plate acetylcholinesterase deficiency. Ann. Neurol. 47; 162-170.

18. Pope, C., Karanth, S. and Liu, J. (2005) Pharmacology and toxicology of cholinesterase inhibitors: uses and misuses of a common mechanism of action. Environ. Toxicol. Pharmacol. 19; 433-446.

19. Pregelj, P. and Sketelj, J. (2002) Role of load bearing in acetylcholinesterase regulation in rat skeletal muscles. J. Neurosci. Res. 67; 114-121.

20. Rieger, F., Koenig, J. and Vigny, M. (1980) Spontaneous contractile activity and the presence of the $16 \mathrm{~s}$ form of acetylcholinesterase in rat muscle-cells in culture. Reversible suppressive action of tetrodotoxin. Dev. Biol. 76; 358-365.

21. Sketelj, J., Crne-Finderle, N., Strukelj, B., Trontelj, J. V. and Pette, D. (1998) Acetylcholinesterase mRNA level and synaptic activity in rat muscles depend on nerve-induced pattern of muscle activation. J. Neurosci. 18; 1944-1952.

22. Soreq, H. and Seidman, S. (2001) Acetylcholinesterase-new roles for an old actor. Nat. Rev. Neurosci. 2; 294-302.

23. Vander, K. W. and Molgo, J. (1994) Quantal acetylcholine release at the vertebrate neuromuscular junction. Physiol. Rev. 74; 899991.

24. Vexler, Z. S., Wong, A., Francisco, C., Manabat, C., Christen, S., Tauber, M., Ferriero, D. M. and Gregory, G. (2003) Fructose-1,6bisphosphate preserves intracellular glutathione and protects cortical neurons against oxidative stress. Brain Res. 17; 90-98.

25. Vignaud, A., Fougerousse, F., Mouisel, E., Guerchet, N., Hourde, C., Bacou, F., Butler-Browne, G. S., Chatonnet, A. and Ferry, A. (2008) Genetic inactivation of acetylcholinesterase causes functional and structural impairment of mouse soleus muscles. Cell Tissue Res. 333; 289-296.

26. Xie, W., Stribley, J. A., Chatonnet, A., Wilder, P. J., Rizzino, A., Mccomb, R. D., Taylor, P., Hinrichs, S. H. and Lockridge, O. (2000) Postnatal developmental delay and supersensitivity to organophosphate in gene-targeted mice lacking acetylcholinesterase. J. Pharmacol. Exp. Ther. 293; 896-902.

This is an open access article distributed under the Creative Commons Attribution License, which permits unrestricted use, distribution, and reproduction in any medium, provided the original work is properly cited. 